\title{
Nongenetic Inheritance of Induced Resistance in a Wild Annual Plant
}

\author{
Åsa Lankinen, Kibrom B. Abreha, Erik Alexandersson, Stefan Andersson, and Erik Andreasson
}

First, second, third, and fifth authors: Swedish University of Agricultural Sciences, Plant Protection Biology, P.O. Box 102, S-230 53 Alnarp, Sweden; and fourth author: Department of Biology, Lund University, Ecology Building, S-223 62 Lund, Sweden. Accepted for publication 4 April 2016.

\begin{abstract}
Lankinen, Å., Abreha, K. B., Alexandersson, E., Andersson, S., and Andreasson, E. 2016. Nongenetic inheritance of induced resistance in a wild annual plant. Phytopathology 106:877-883.

Nongenetic inheritance (e.g., transgenerational epigenetic effects) has received increasing interest in recent years, particularly in plants. However, most studies have involved a few model species and relatively little is known about wild species in these respects. We investigated transgenerational induced resistance to infection by the devastating oomycete Phytophthora infestans in Solanum physalifolium, a wild relative of cultivated potato. We treated plants with $\beta$-aminobutyric acid (BABA), a nontoxic compound acting as an inducing agent, or infected plants with $P$. infestans.

BABA treatment reduced lesion size in detached-leaf assays inoculated by $P$. infestans in two of three tested genotypes, suggesting that resistance to oomycetes can be induced by BABA within a generation not only in crops or model species but also in wild species directly collected from nature. Both BABA treatment and infection in the parental generation reduced lesions in the subsequent generation in one of two genotypes, indicating a transgenerational influence on resistance that varies among genotypes. We did not detect treatment effects on seed traits, indicating the involvement of a mechanism unrelated to maternal effects. In conclusion, our study provides data on BABA induction and nongenetic inheritance of induced resistance in a wild relative of cultivated potato, implying that this factor might be important in the ecological and agricultural landscape.
\end{abstract}

Nongenetic inheritance is a collective name of various inheritance mechanisms mediated by the transmission to offspring of elements of the parental phenotype or environment, including epigenetic marks and other parental influence (Bonasio 2015; Bonduriansky and Day 2009; Danchin et al. 2011). Here, alternative inheritance factors influence the offspring directly and alongside Mendelian heredity (i.e., transfer of gene alleles [DNA sequence variants]) (Bonduriansky 2012). Nongenetic inheritance, particularly epigenetic changes (including DNA methylation, histone modifications, and small, noncoding RNA), has received an increasing interest in the past 5 to 10 years (Kawashima and Berger 2011; Sharma 2013). However, the conceptual papers outnumber the relatively few experimental studies that have been conducted.

To date, evidence for transgenerational epigenetic inheritance exists in several taxa, particularly in unicellular organisms, fungi, and plants (Heard and Martienssen 2014; Jablonka and Raz 2009; Van Oosten et al. 2014). It is clear that some of the transgenerational epigenetic effects are nonadaptive, such as when they cause disease in humans (Burggren and Crews 2014; Youngson and Whitelaw 2008). It has also been suggested that they can be adaptive by mediating phenotypic plasticity and rapid genetic adaptation in a variable environment (Bonduriansky and Day 2009; Bonduriansky et al. 2011; Danchin et al. 2011; Shea et al. 2011). Recent mathematical models show that evolution of nongenetic inheritance is affected by environmental factors, the level of gene flow, and the mechanism underlying nongenetic inheritance (English et al. 2015; Leimar and McNamara 2015). However, to understand the adaptive basis of transgenerational epigenetics, more empirical data are needed, particularly on wild species from natural environments (Becker and Weigel 2012; Richards 2011).

In plants, it is known that abiotic and biotic factors can cause transgenerational epigenetic changes, such as DNA methylation

Corresponding author: ^̊. Lankinen; E-mail address: asa.lankinen@slu.se
(Van Oosten et al. 2014; Yu et al. 2013). Environmentally induced DNA methylation typically lasts two to five or even up to at least eight generations for $50 \%$ of the methylatable sites (Johannes et al. 2009; Reinders et al. 2009; Teixeira et al. 2009). Even though transgenerational induction of resistance to herbivores or pathogens has been detected in several species (Henry et al. 2013; Holeski et al. 2012), most studies have been performed on model species (e.g., Arabidopsis thaliana). However, in wild Taraxacum officinale, herbivory showed heritable effects on offspring that varied among genotypes (Verhoeven and van Gurp 2012). Moreover, in a longterm investigation of Viola cazorlensis in its natural habitat, Herrera and Bazaga (2011) separated the environmental, genetic, and epigenetic components of phenotypic variation and found that epigenetic variation was correlated with herbivore damage.

Plant resistance to natural enemies can be induced by biotic and abiotic agents. Such triggering of the immune response can also increase the level of responsiveness to later arriving pathogens by a faster or stronger resistance reaction, referred to as induced resistance (Bengtsson et al. 2014a) or priming of defense (Pastor et al. 2013). These responses include both short-lasting and longlasting effects, where the latter include transgenerational effects caused by DNA methylation (Luna et al. 2012; Yu et al. 2013). A. thaliana exposed to an avirulent strain of Pseudomonas syringae, or inducing treatments with the chemical $\beta$-aminobutyric acid (BABA), showed enhanced resistance to infection by virulent strains of $P$. syringae and Hyaloperonospora arabidopsidis in the offspring generation (Slaughter et al. 2012). Moreover, an additional inducing treatment of the offspring generation resulted in an even stronger resistance reaction in the following generation, suggesting nongenetic inheritance of the ability to induce resistance. We are not aware of studies in which BABA-induced resistance, or its possible transgenerational effects, have been tested in plant material collected in the wild, which is crucial for our understanding of the importance of nongenetic inheritance under natural conditions. BABA is one of the most studied elicitors of induced resistance (Bengtsson et al. 2014b; Liljeroth et al. 2010; Luna et al. 2014). Learning more about epigenetic inheritance of 
factors that influence plant disease could be significant for developing breeding material of crops using novel resistance factors (Zhang and Hsieh 2013), or improving our understanding of factors affecting the presence of pathogens in the ecological and agricultural landscape.

It should be noted that provisioning of seed could be negatively influenced by induced resistance, as has been shown in some species (Walters and Heil 2007). Moreover, BABA treatment of seed has been shown to increase abiotic stress tolerance in seedlings (Jisha and Puthur 2016). Maternal effects mediated though differential seed provisioning may be nongenetic (Bonduriansky and Day 2009; Roach and Wulff 1987) but are generally considered distinct from epigenetic influences (Holeski et al. 2012).

The overall aim of the present study was to investigate the presence of induced resistance to infection and potential influence of nongenetic inheritance in Solanum physalifolium Rusby, a wild relative of cultivated potato not subjected to artificial selection (unlike, for example, research in Arabidopsis). The species is invasive in southern Sweden and has been considered as a serious weed in agricultural fields with open soil (Grönberg et al. 2012). $S$. physalifolium is highly susceptible to infection by the oomycete Phytophthora infestans (Mont.) de Bary, the pathogen causing late blight in cultivated potato. In fact, $S$. physalifolium has been reported to be more susceptible to $P$. infestans than many cultivated potato cultivars and, therefore, may change the population structure of this pathogen (Frades et al. 2015; Grönberg et al. 2012). We performed a 3-year greenhouse study in which wild-collected plants were treated with BABA, infected by the pathogen $P$. infestans, and then evaluated with respect to resistance to $P$. infestans and seed traits as a measure of fitness cost of the induced resistance (relative to a control group). Even though BABA can induce resistance in a large number of cultivated plants, no previous study has investigated such effects in wild-collected plants and looked at their transgenerational consequences. We asked five questions. (i) Can BABA induce resistance against $P$. infestans in wild-collected $S$. physalifolium within the same plant generation? (ii) Is the BABA-induced resistance transgenerational? (iii) Do we find a similar transgenerational effect following infection by $P$. infestans? (iv) Is the BABAinduced transgenerational effect increasing after two generations of
BABA treatments? (v) Is the transgenerational effect of induced resistance related to plant size or seed number and size (i.e., is there evidence for maternal provisioning of seed or cost of resistance?).

\section{MATERIALS AND METHODS}

Plant material. Hairy nightshade (S. physalifolium Rusby) is a diploid annual weed native to Argentina, Bolivia, and Chile. Of the two described varieties of this species, the more common one, S. nitidibaccatum (Bitter) Edmonds (Edmonds 1986), is widely naturalized in Europe, Australia, New Zealand, Canada, and the United States. Plant material for the present study was collected as seed from three natural populations growing in agricultural fields with row crops within a 5-km stretch along the southern Swedish coast (Lillgårda farm, Löderup, $55.39^{\circ} \mathrm{N}, 14.13^{\circ} \mathrm{E}$; Stockholmsgården farm, Valleberga, $55.42^{\circ} \mathrm{N}, 14.08^{\circ} \mathrm{E}$; and KLM farm, Kåseberga, $55.39^{\circ} \mathrm{N}, 14.09^{\circ} \mathrm{E}$; denoted I, L, and $\mathrm{K}$, respectively). We used one genotype per population in order to ensure high genetic diversity in our experiment.

Seed germination and clonal propagation of plants. To prepare for germination, seed were washed with $\mathrm{EtOH}(70 \%)$ for $1 \mathrm{~min}$, soaked in a chlorine solution (1\%) for $5 \mathrm{~min}$, and rinsed three times with sterile MilliQ water. Clean seed were placed in a petri dish with a filter paper saturated with growth medium (gibberellin acid-3 at $0.5 \mathrm{~g} \mathrm{ml}^{-1}$ and $\mathrm{KNO}_{3}$ at $1 \mathrm{~g} \mathrm{ml}^{-1}$; Duchefa, Haarlem, The Netherlands) diluted in $0.5 \mathrm{ml}$ of tap water. Parafilm-sealed petri dishes were incubated in a growth chamber conditioned to $16 \mathrm{~h}$ light and $8 \mathrm{~h}$ of darkness, 22 and $19^{\circ} \mathrm{C}$ maximum and minimum temperatures, $65 \%$ relative humidity, and light intensity of $180 \mu \mathrm{mol} \mathrm{m} \mathrm{m}^{-2} \mathrm{~s}^{-1}$. Seedlings were transferred to pot soil 2 weeks after sowing and grown in the growth chamber as described by Bengtsson et al. (2014a).

To obtain genetically identical clones, we took single-node stem cuttings ( 20 to $30 \mathrm{~mm}$ ) from three plants (one from each population, hereafter called genotypes) at the age of 6 weeks and placed them on wet soil covered with plastic bags for 5 days. Five to six replicate clones were obtained from each of the three genotypes, hereafter referred to as parental generation (Parental-2012: I, K, and L) (Fig. 1) and grown for 4 weeks before starting the experimental
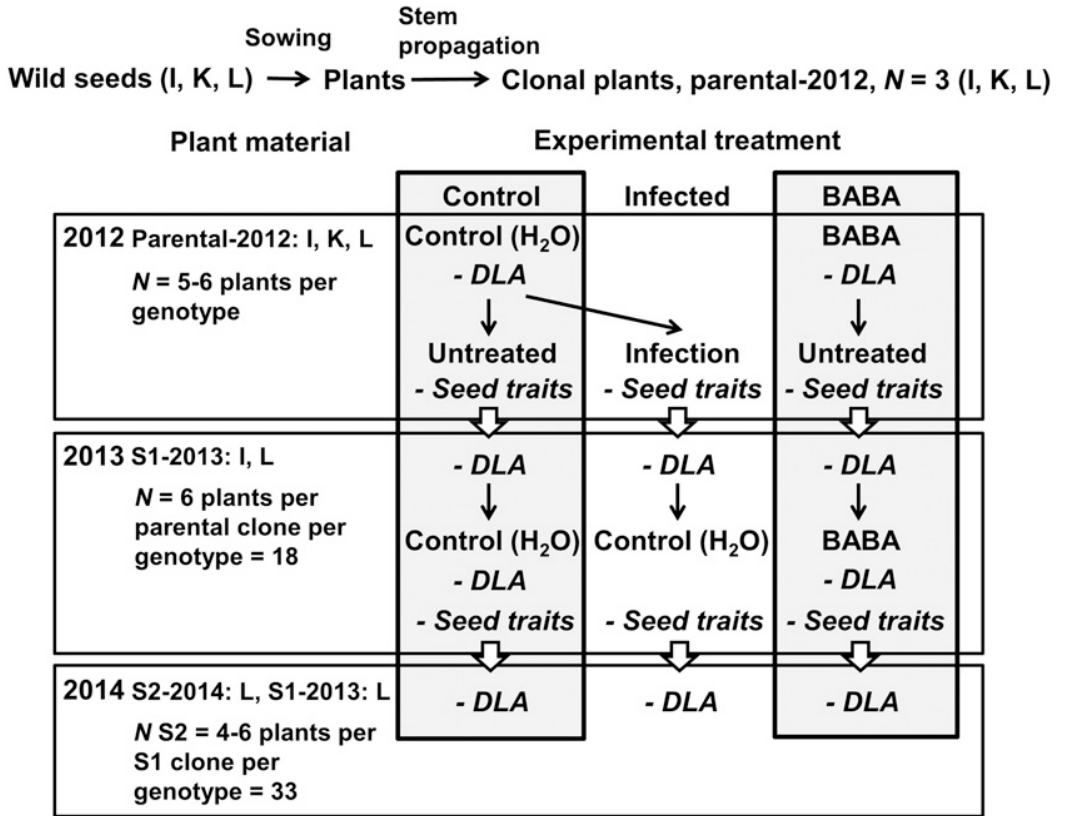

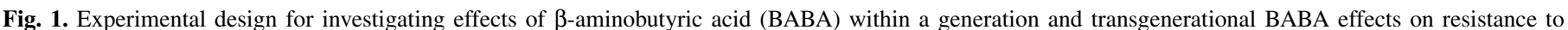

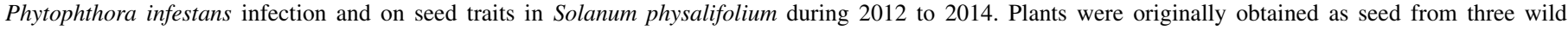

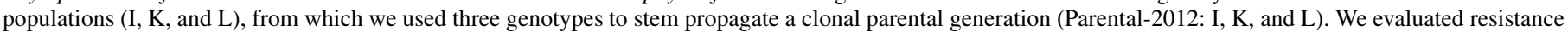

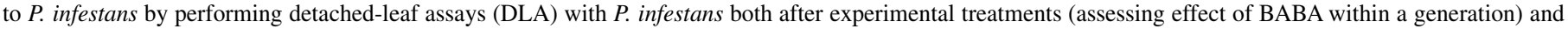
prior to treatments (assessing transgenerational effects of BABA on infection). 
treatments. At this time, plants had started to flower and, occasionally, some flowers had developed into small, unripe berries.

Establishment of experimental generations. To study effects of induced resistance to infection and nongenetic inheritance, we generated experimental generations during 2012 to 2014 (parental, S1 = first offspring generation and S2 = second offspring generation) that were (i) BABA treated in 2012 to 2013, (ii) infected with $P$. infestans in 2012, or (iii) used as controls (Fig. 1). In 2012, plants of the parental generation ( $n=5$ to 6 per genotype) were either foliar treated with BABA $(10 \mathrm{mmol}, 10 \mathrm{ml} / \mathrm{plant}$, three times with a 4- to 5-day interval) or the same amount of water (control) (Fig. 1). The amount and frequency of BABA added was based on previous studies in cultivated potato (Liljeroth et al. 2010). In addition to the two main treatments (BABA and control), approximately half of plants in the control group $(n=2)$ were whole plant infected, allowing us to investigate induced resistance in relation to infection. For the whole-plant infections 10 intact leaves per clone were drop inoculated with a suspension of $P$. infestans strain 88069 of 15,000 sporangia $\mathrm{ml}^{-1}$ and plants were incubated in the infection chamber as described by Abreha et al. (2015). Inoculated leaves were removed when showing disease symptoms, because the infection was expanding into the noninoculated leaves and the plants had to survive to produce seed for the next generation.

In 2013 and 2014, we followed the offspring from the previous years, generating $\mathrm{S} 1$ ( $n=6$ per maternal plant) and $\mathrm{S} 2$ ( $n=4$ to 6 per maternal plant), respectively (Fig. 1). S1 plants previously treated with BABA were treated again with BABA at the same age and developmental stage as in the previous year using the same technique. S1 included the two genotypes where we had detected an effect of BABA within the parental generation (I and L) (see Results section). S2 included the L genotype, where we had found a transgenerational effect in 2013 (see Results section). In both years, seed were sown, seedlings were transferred to soil, and plants were grown in the growth chamber as described above.

In 2012 and 2013, we collected ripe berries 4 weeks after initial experimental treatments. At the time of experimental treatments, branches of both BABA-treated and untreated plants were marked with a thread to locate berries that had been flowers at the time of the experimental treatment in order to collect seed that had been influenced by treatment. Seed were extracted from the berries, cleaned, air dried, and then stored in a freezer $\left(-20^{\circ} \mathrm{C}\right)$ until the next summer.

Resistance bioassays and estimates of plant size and seed traits. We used detached-leaf assays (DLA) to attest to P. infestans resistance differences between experimental treatments both after BABA treatments (effect of BABA within a generation) in 2012 and 2013 and before BABA treatments (transgenerational BABA effect) in 2013 and 2014 (Fig. 1). The DLA were performed 2 days after the final BABA treatment, following the procedure described for potato (Liljeroth et al. 2010), on 6-week-old plants which had started flowering. We drop inoculated eight detached leaves per clone with a suspension of $P$. infestans strain SE-03058 of 15,000 sporangia $\mathrm{ml}^{-1}$ (Ali et al. 2012). Response to infection by P. infestans was estimated as the area of developed lesions at 7 days postinfection (dpi), calculated as the length of the longest axis multiplied by the maximum width perpendicular to that axis. As a control for DLA and infectivity of the inoculum used, we always conducted parallel DLA on sensitive 'Desirée' potato $(n=6)$.

To ensure that we distributed plants of equal size to experimental treatments in the parental generation and allowed testing for differences in size between the three genotypes, we measured plant size as plant height times number of branches 1 day before DLA. To investigate effects of maternal provisioning of seed on experimental treatments or a possible indication of a cost of increased ability to induce resistance, we counted and weighed the collected and airdried seed from 5 berries/plant. We analyzed the traits of weight per berry and weight per seed but not number of seed per berry because this trait would be redundant in relation to the other traits.
Estimates of plant size under field conditions. To evaluate whether the genotypic differences in plant size (see Results section) were maintained under field conditions, plants of the I and L genotypes were grown outdoors in 2013. In all, 24 seedlings per genotype were planted in four blocks in an experimental garden at SLU Alnarp, Sweden. Plant size was measured at the age of 4 and 7 weeks as the product of two maximum plant width estimates (perpendicular to each other), representing plant coverage area.

Data analysis. Effect of BABA treatment within a generation on lesion size at 7 dpi in 2012 and 2013 was tested by pairwise comparisons between treatments in each of the three genotypes of the parental generation and the two genotypes of the S1 generation using a nonparametric method (Kruskal-Wallis) because data were not normally distributed (many zeros). Similar tests were used to investigate differences between year 2012 and 2013 for each treatment and genotype. In all tests involving lesion size as the dependent variable, we pooled all leaves from the different plants within a genotype and treatment. Variability within a plant, estimated using the coefficient of variation, was, on average, approximately $20 \%$.

Analyses of the dependent variables lesion size in bioassays performed early in the season (to explore presence of a transgenerational BABA effect), plant size, and seed traits were conducted with a series of two-way analyses of variance (ANOVA) in SPSS (SPSS 2014), because these variables did not include zeros. Depending on the specific question asked, we used the following combination of independent variables (all treated as fixed factors): (i) treatment, genotype of paternal generation, and interaction (data from 2012); (ii) treatment, genotype of S1 generation, and interaction (data from 2013); (iii) treatment, year of evaluating S1 generation, and interaction (data from 2013-2014); and (iv) treatment, generation (S1 versus S2), and interaction (data from 2014). A significant difference between control groups within analyses iii and iv would suggest an environmental influence. To test for differences in plant size under field conditions, the ANOVA model involved genotype and experimental block. Because of the low number of genotypes included in our study, we considered the factor genotype to be fixed rather than random. To further explore significant differences between factors, we performed a posthoc test (Tukey's highly significant difference [HSD]), either following the two-way ANOVA (when the interaction was unimportant) or following complementary one-way ANOVA, in which each combination of treatments was treated as a separate group. For analyses of seed traits in 2012, we pooled data for control and infected plants, because $n$ values did not allow testing infected plants separately and the whole-plant infections were performed much later than BABA treatments (when the seed we collected had already been formed).

We used type III sum of squares in all ANOVA and all models were validated with respect to normality of residuals and homogeneity of variances. Weight per berry and weight per seed were squareroot transformed in 2012 but not in 2013, to obtain normally distributed residuals.

\section{RESULTS}

Effect of BABA treatment within a generation. In 2012, plants pretreated with BABA in the same year (within the same generation) showed reduced lesion size in DLA of $P$. infestans infection in the parental generation for the genotypes I and $\mathrm{L}$ but not K (Kruskal-Wallis, I: $\chi^{2}=6.67$, df $=1, n=40, P=0.010$; L: $\chi^{2}=21.3, \mathrm{df}=1, n=48, P<0.001$; and $\mathrm{K}: \chi^{2}=0.52$, df $=1, n=48$, $P=0.47$; Fig. 2A). An effect of direct BABA treatment was also seen in 2013 in the next generation (S1) in both tested genotypes (I and L) (Kruskal-Wallis, I: $\chi^{2}=7.29$, $\mathrm{df}=1, n=70, P=0.007$; and L: $\chi^{2}=7.51$, df $=1, n=60, P=0.006$; Fig. $2 \mathrm{~B}$ ). There were no significant differences between years for any of the treatments (Kruskal-Wallis, $P>0.11$ ). 
The susceptible potato cultivar Desiree acting as a control for DLA on S. physalifolium showed a strong response to infection by $P$. infestans as expected in both years (lesion size \pm standard deviation [SD], 2012: $524 \pm 96.6 \mathrm{~mm}^{2}, n=4 ; 2013: 406 \pm 168 \mathrm{~mm}^{2}$, $n=15 ; t$ test; $P=0.20$ ).

Plant size of genotypes in parental generation, measured as plant height times number of branches at the same time as DLA but before inoculation, did not differ between BABA and control treatment (two-way ANOVA, treatment: $F_{1,11}=1.13, P=0.31$; genotype: $F_{2,11}=7.21, P=0.010$; treatment-genotype: $F_{2,11}=0.133$, $P=0.87$ ). Genotype $\mathrm{L}$ was smaller than the other genotypes (mean difference, Tukey's HSD: $\mathrm{L}$ to $\mathrm{I}=-3,380 \mathrm{~mm}, P=0.011$; $\mathrm{L}$ to $\mathrm{K}=$ $-2,500 \mathrm{~mm}, P=0.045)$, while the other genotypes did not differ in size ( $\mathrm{I}$ to $\mathrm{K}=890 \mathrm{~mm}, P=0.63$ ).

Transgenerational effect of induced resistance. To test for a transgenerational effect of induced resistance by BABA or infection by $P$. infestans on lesion size, we compared the detachedleaf assays from I and L genotypes in 2013 in the first offspring generation (S1). We found that lesion size was strongly affected by the interaction between treatment and genotype; that is, the two genotypes responded differently to the treatments (two-way ANOVA, treatment: $F_{2,174}=12.3, P<0.001$; genotype: $F_{1,174}=7.00$, $P=0.009$; treatment-genotype: $\left.F_{2,174}=11.1, P<0.001\right)$. Analysis of each of the three treatments per genotype using posthoc tests showed that, in the L genotype, control plants had larger lesions than in the other two treatments (BABA or P. infestans infection)
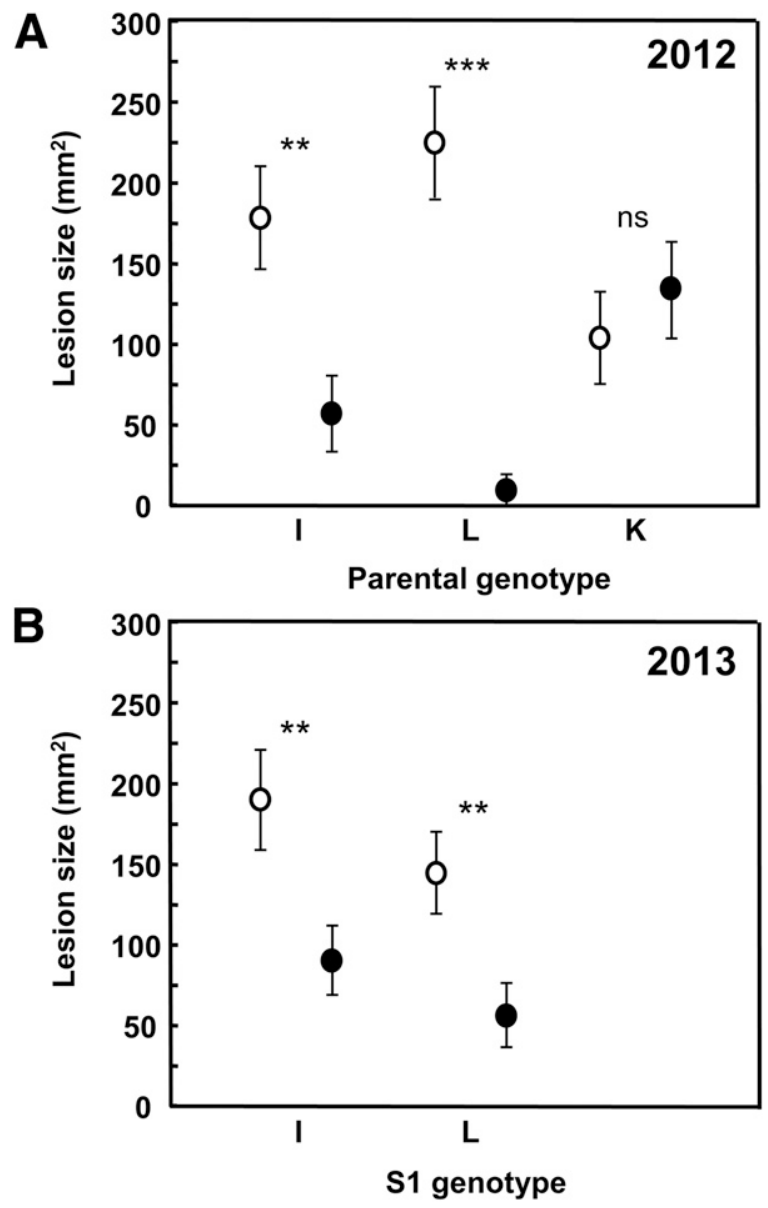

Fig. 2. Lesion size in Solanum physalifolium as an effect of $\beta$-aminobutyric acid (BABA) treatment within a generation (i.e., in the same year [filled circles] or control [open circles]) on Phytophthora infestans infection on $\mathbf{A}$, genotypes I, L, and K of parental generation in 2012 and B, genotypes I and $\mathrm{L}$ of first offspring generation (S1) in 2013. Bioassays were performed on detached leaves and area of developed lesions was measured 7 days postinfection. Error bars indicate standard errors; $\mathrm{ns}=P>0.05$, ** $=P<0.01$, and $* * *=P<0.001$ in pairwise comparisons.
(Fig. 3). Thus, in the L genotype, treatments applied the previous year (BABA or $P$. infestans infection) resulted in smaller lesions, which is the expected pattern for a transgenerational effect. This pattern was not seen in the I genotype (Fig. 3).

To further confirm the transgenerational treatment effect of induced resistance in the L genotype of the S1 generation in 2013, we evaluated this genotype also in 2014. In an analysis of the L genotype in both 2013 and 2014, we found that lesions were generally smaller in the second year (mean \pm standard error, 2013: $549 \pm 17.4 \mathrm{~mm}^{2}$ and 2014: $\left.463 \pm 15.8 \mathrm{~mm}^{2}\right)$, but the treatment effect was consistent between years (two-way ANOVA, treatment: $F_{2,180}=$ $30.9, P<0.001$; year: $F_{1,180}=16.4, P<0.001$; treatment-year: $\left.F_{2,180}=0.956, P=0.39\right)$. The averaged data for 2013 and 2014 suggested that infection with $P$. infestans in the previous year did not reduce lesions as much as BABA treatment alone (one-way ANOVA, treatment: $F_{2,183}=28.1, P<0.001$; mean difference, Tukey's HSD, control-BABA: $189 \mathrm{~mm}^{2}, P<0.001$; control-infected: $78.4 \mathrm{~mm}^{2}$, $P=0.010$; BABA-infected: $\left.-110 \mathrm{~mm}^{2}, P<0.001\right)$.

In contrast to the year effect detected on lesion size in the L genotype of the $\mathrm{S} 1$ generation, lesion size of the control susceptible Desiree potato did not differ between the two experimental years (lesion size $\pm \mathrm{SD}, 2013: 560 \pm 81.7 \mathrm{~mm}^{2}, n=30 ; 2014: 598 \pm$ $136 \mathrm{~mm}^{2}, n=6$; $t$ test, $P=0.51$ ).

Effect of generation on transgenerational induced resistance. Evaluation of the L genotype of the S1 and S2 generations, both grown in 2014, revealed significant effects of experimental treatment and generation on lesion size but no significant interaction between these two factors (two-way ANOVA, treatment: $F_{2,191}=$ 30.4, $P<0.001$; generation: $F_{1,191}=4.01, P=0.047$; treatmentgeneration: $\left.F_{2,191}=2.86, P=0.060\right)$. Thus, despite smaller lesions in the second generation, BABA treatment still reduced lesions compared with control over both generations, confirming a transgenerational effect of BABA added in the previous year (Fig. 4). In contrast, the infection treatment added in 2012 did not reduce lesion size compared with control in the second generation (Fig. 4), suggesting that the transgenerational effect of infection was unstable over time.

Including multiple generations allowed us to ask whether there was an increasing transgenerational effect in the S2 generation,

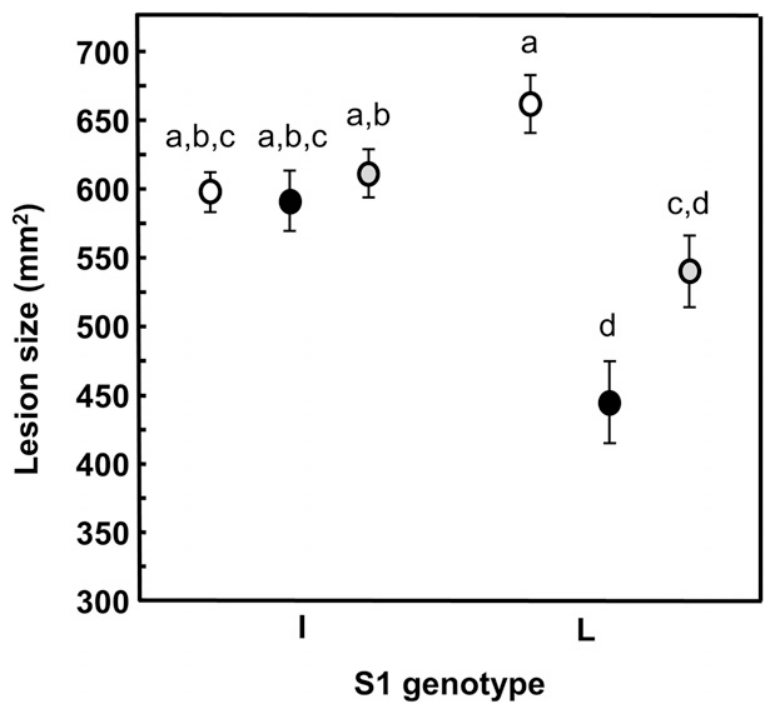

Fig. 3. Lesion size in Solanum physalifolium evaluated in 2013 as an effect of $\beta$-aminobutyric acid (BABA) (filled black circles), infection (filled gray circles), or control (open circles) treatment applied in the previous year (2012) on Phytophthora infestans infection in genotypes I and L of the first offspring generation (S1). Bioassays were performed on detached leaves and area of developed lesions was measured 7 days postinfection. Error bars indicate standard errors. Different letters indicate a significant difference in a posthoc test (Tukey's highly significant difference following one-way analysis of variance; treatment: $\left.F_{5,174}=10.1, P<0.001\right)$. 
where plants had been BABA-treated over two consecutive years compared with in the $\mathrm{S} 1$ generation, with only one previous BABA treatment. A separate analysis of control and BABA treatment could not detect a significant interaction between treatment and generation, as would be expected from an accelerating effect, thus indicating that the transgenerational BABA effect was consistent between generations (two-way ANOVA, treatment: $F_{1,139}=40.7$, $P<0.001$; generation: $F_{1,139}=8.39, P=0.004$; treatmentgeneration: $F_{1,139}=0.739, P=0.39$ ) (Fig. 4).

Seed traits in relation to induced resistance-treatments. Weight of berries and weight per seed did not differ between BABA versus the combination of control and infection treatments in 2012 (two-way ANOVA, berry weight: treatment: $F_{1,11}=1.10, P=0.32$; parental genotype: $F_{2,11}=11.7, P=0.002$; treatment-parental genotype: $F_{2,11}=1.03, P=0.39$; weight per seed: treatment: $F_{1,11}=$ $0.011, P=0.92$; parental genotype: $F_{2,11}=0.322, P=0.73$; treatment-parental genotype: $F_{2,11}=1.33, P=0.30$ ) or between the three experimental treatments in 2013 (two-way ANOVA, berry weight: treatment: $F_{2,30}=0.097, P=0.91$; S1 genotype: $F_{1,30}=$ $0.378, P=0.54$; treatment-S1 genotype: $F_{2,30}=0.269, P=0.77$; weight per seed: treatment: $F_{2,30}=0.725, P=0.49$; S1 genotype: $F_{1,30}=1.09, P=0.31$; treatment-S1 genotype: $F_{2,30}=0.548, P=$ $0.58)$. In 2012 , the $\mathrm{K}$ genotype had higher berry weight than the other genotypes (mean difference, Tukey's HSD, K to I: $23.4 \mathrm{mg}$, $P<0.003$; K to L: $12.5 \mathrm{mg}, P=0.015$; I to $\mathrm{L}:-1.69 \mathrm{mg}, P=0.82$ ).

Plant size under field conditions. The I and L genotypes grown under field conditions in 2013 differed in plant size measured as plant coverage area at both 4 and 7 weeks of age (two-way ANOVA, 4 weeks: genotype: $F_{1,43}=9.91, P=0.003$; block: $F_{3,43}=$ $2.51, P=0.072$; 7 weeks: genotype: $F_{1,43}=22.1, P<0.001$; block: $\left.F_{3,43}=0.982, P=0.41\right)$. Consistent with the greenhouse results, $\mathrm{L}$ plants were smaller than I plants (mean difference $\mathrm{L}$ to $\mathrm{I}=-0.058$ and $-1.20 \mathrm{~m}^{2}$ at 4 and 7 weeks, respectively).

\section{DISCUSSION}

In this study of $S$. physalifolium, we found that pretreatment with BABA reduced symptoms of $P$. infestans infection in two of three investigated genotypes. In the next generation, one genotype still showed an effect of previous BABA treatment, indicating a transgenerational effect on plant resistance. An additional BABA treatment in the offspring generation showed a similar transgenerational effect on resistance in a second offspring generation, although the inducibility of resistance did not increase with an added treatment. Infection of the same pathogen was also shown to give a transgenerational effect of reduction of disease symptoms, allowing for the possibility that induced resistance can have an effect in the ecological and agricultural landscape.

BABA and induced resistance against $P$. infestans infection in a wild species. Effects of BABA treatments on induced resistance to biotic agents have previously been shown to be effective in at least 14 plant families and 37 species (Bengtsson 2013). However, the majority of these are crops, including cultivated potato (Liljeroth et al. 2010), where BABA treatment also increased resistance to $P$. infestans in the next vegetative progeny (FloryszakWieczorek et al. 2015). There is no previous report studying response to pathogens in wild plant material. In the present study, we investigated resistance induction in a wild species, S. physalifolium, a relative of cultivated potato, using seed directly collected in the wild. BABA treatment reduced the development of symptoms caused by $P$. infestans in $S$. physalifolium in DLA (effect of BABA within a generation) but the effects were genotypespecific: two of the three investigated genotypes showed a detectable response. It is possible that all genotypes would have responded to BABA if we had used additional treatments or a higher dosage but our results still suggest a difference in the ability to respond to BABA. In potato, inducibility has been shown to differ between potato cultivars (Liljeroth et al. 2010), with a tendency for partially resistant cultivars to show more induced resistance. In contrast, our results indicate that the most resistant genotype $(\mathrm{K})$ showed the lowest response to BABA; thus, we could hypothesize a trade-off between the ability to respond to inductive compounds and the basal level of resistance response reducing growth. Plant size of genotypes of the parental generation differed, and the difference between L and I progeny was confirmed in the field. However, because $\mathrm{L}$ plants were smaller than the other genotypes ( $\mathrm{I}$ and $\mathrm{K}$ ), there is no clear indication that plant size covaries with the magnitude of response to BABA (I and L). We cannot, however, rule out the possibility that differences in BABA responsiveness reflect differences in surface structure and chemistry of leaves (Balmer et al. 2015; Pastor et al. 2014).

Transgenerational effects on plant resistance against P. infestans. The offspring of the two genotypes that responded to BABA treatment in the parental generation (I and L) showed contrasting responses in DLA to $P$. infestans infection; lesion size was still reduced in the L but not in the I genotype. Thus, the BABAinduced resistance response was found to be transgenerational in one of two evaluated genotypes. Unlike previous studies that found transgenerational effects on plant resistance to pathogens (Bilichak et al. 2015; Kathiria et al. 2010; Luna et al. 2012; Mandal et al. 2012; Slaughter et al. 2012; Walters and Paterson 2012), we used wild plant material collected directly from natural populations with exposure to a complex set of stressors, including $P$. infestans, but with no history of artificial selection. Interestingly, the genotypic differences detected both for the BABA effect within a generation and the transgenerational BABA effect suggest that we have variability regarding induced resistance both within and between generations. Because our genotypes came from different localities, we hypothesize that these differences are driven by environmental factors (Leimar and McNamara 2015). However, because we did not sample multiple genotypes from each population, we do not know if the detected differences are consistent among populations or if similar variability exists within populations. Variability can also be generated when genetic and epigenetic factors interact (Bonduriansky and Day 2009; Herrera et al. 2014) or because of clockwise accumulation of mutations (i.e., generating selectively neutral genetic variation) (Hagmann et al. 2015). In future studies in S. physalifolium, it would be of great interest to quantify variation in

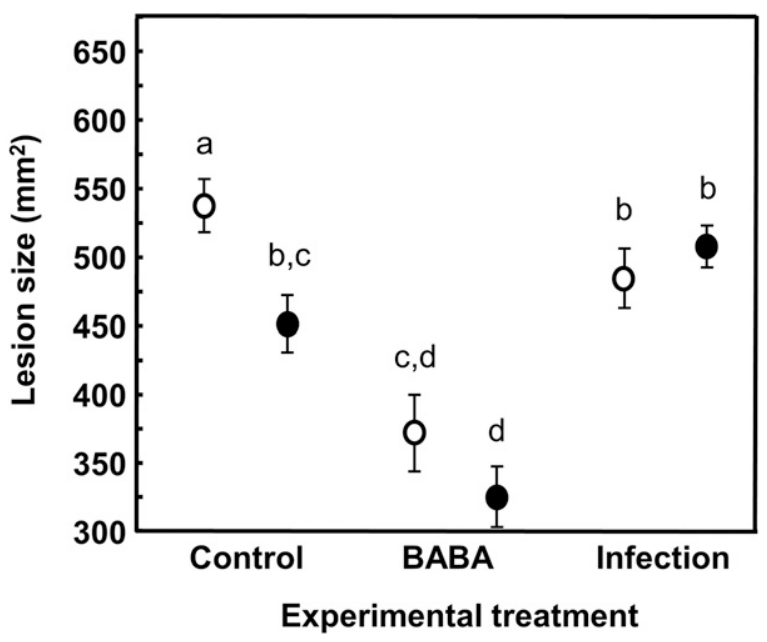

Fig. 4. Lesion size in Solanum physalifolium evaluated in 2014 as an effect of $\beta$-aminobutyric acid (BABA), infection, or control treatment applied in one (S1 generation, open circles) or two (S2 generation, filled circles) previous generations on Phytophthora infestans infection in the L genotype. Bioassays were performed on detached leaves and area of developed lesions was measured 7 days postinfection. Error bars indicate standard errors. Different letters indicate a significant difference in a posthoc test (Tukey's highly significant difference following one-way analysis of variance; treatment: $F_{5,181}=23.6$, $P<0.001)$ 
induced resistance and its epigenetic influence within and between multiple populations.

In the present study, we also performed an infection treatment with $P$. infestans in the parental generation. The inoculation treatment resulted in a transgenerational response in the offspring similar to that in the BABA-treated plants. However, the response was more pronounced for the BABA treatment. This is in agreement with other studies showing that inducing resistance with chemicals gives a more consistent and less variable result compared with biotic agents (Pastor et al. 2013). In A. thaliana, inoculation with virulent Pseudomonas syringae also showed a transgenerational effect on induced resistance (Luna et al. 2012) but this effect was not compared with that of BABA induction. Moreover, our results in S. physalifolium suggest that induced resistance could be a factor in the ecological and agricultural landscape where Phytophthora infestans spores are present.

Effects of experimental treatments on plant resistance in multiple generations. In A. thaliana, it has previously been shown that repeated BABA treatment increased the transgenerational effect on induced resistance toward bacteria (Slaughter et al. 2012). In the present study, the transgenerational BABA effect did not increase when plants were induced in two previous generations compared with in one previous generation. We did observe differences between control groups for the different generations, which presumably were caused by some environmental factor (e.g., longer storage of seed for the first offspring generation). The difference between experimental results from $A$. thaliana and $S$. physalifolium may be attributed to differences in species-specific factors, in the origin of the plant material (lab versus wild populations), or in the behavior of the pathogen. We believe that using wild plant material rather than artificially selected greenhouse material gives a more reliable picture of processes in natural environments regarding the effects of repeated treatment. Moreover, at present, it is difficult to imagine that a constantly increasing inducibility would be beneficial in nature. Additional research on wild plant materials grown in their natural environment in the presence of complex biotic and abiotic stressors would allow for a better understanding of the importance of inducibility in agroecosystems.

Unlike the BABA treatment, we only inoculated plants with $P$. infestans in the first generation. We were unable to show a transgenerational effect in the second offspring generation for the infection treatment, suggesting that this effect levels off relatively fast. Only a few other studies have investigated transgenerational effects on induced resistance to pathogens for more than the parental and one offspring generation. In Nicotiana tabacum, the effect lasted in both investigated offspring generations (Kathiria et al. 2010). A similar result was seen in one Arabidopsis study (Luna et al.2012) but not in another, where the effect lasted in one of the two investigated offspring generations (Slaughter et al. 2012).

Potential mechanisms and cost of transgenerational effect on plant resistance. Previously detected transgenerational effects on plant resistance to pathogens or herbivores suggest involvement of various mechanisms, including epigenetic or maternal effects (Holeski et al. 2012). In the present study, we investigated seed traits to get an indication of whether the observed transgenerational effects could be attributed to maternal effects on seed. There were no differences in seed traits between experimental treatments in either the first or second generation. Thus, we have no evidence that the detected transgenerational effects are a direct consequence of larger or heavier seed. However, we cannot exclude other types of maternal effects (Roach and Wulff 1987). The lack of difference in seed traits between treatments also indicates that BABA treatment did not lead to a detectable cost of resistance. Direct activation of plant resistance is often associated with high allocation costs (van Hulten et al. 2006; Walters and Heil 2007). BABA treatments in crops showed that a high concentration of BABA could confer a cost whereas low concentrations did not, or even increased seed germination and seedling vigor (Nandeeshkumar et al. 2009; Shailasree et al. 2001).
Conclusions. We showed that plant resistance can be activated by BABA not only in crops or model species but also in a wild plant species originating from seed collected in natural populations, which is a novel finding. Our study adds to the growing number of species with detectable nongenetic inheritance and also suggests that transgenerational effects on plant resistance are not unique to cultivated plants or model organisms. In the future, it would be of interest to learn about the underlying mechanisms in our study system. Future studies on nongenetic inheritance should also focus more on wild plants, because this will help us to understand the generality of transgenerational epigenetic effects and whether they can be adaptive. Because a short-term infection of the same pathogen used to assess plant resistance in our study also resulted in a transgenerational effect of reduction of disease symptoms, this underpins the idea that induced resistance might have an effect in the ecological and agricultural landscape.

\section{ACKNOWLEDGMENTS}

We thank L. Andersson and S. Hydbom for collection of seed. This work was supported by PlantLink (to $\AA$. Lankinen, S. Andersson, and E. Andreasson), and the Swedish foundation for strategic research (to E. Andreasson).

\section{LITERATURE CITED}

Abreha, K. B., Alexandersson, E., Vossen, J. H., Anderson, P., and Andreasson, E. 2015. Inoculation of transgenic resistant potato by Phytophthora infestans affects host plant choice of a generalist moth. PLoS One 10:e0129815.

Ali, A., Moushib, L. I., Lenman, M., Levander, F., Olsson, K., Carlson-Nilson, U., Zoteyeva, N., Liljeroth, E., and Andreasson, E. 2012. Paranoid potato: Phytophthora-resistant genotypes shows constitutively activated defense. Plant Signal. Behav. 7:400-408.

Balmer, A., Pastor, V., Gamir, J., Flors, V., and Mauch-Mani, B. 2015. The 'primeome': Towards a holistic approach to priming. Trends Plant Sci. 20:443-452.

Becker, C., and Weigel, D. 2012. Epigenetic variation: Origin and transgenerational inheritance. Curr. Opin. Plant Biol. 15:562-567.

Bengtsson, T. 2013. Boosting potato defence against late blight. Ph.D. thesis, Swedish University of Agricultural Sciences.

Bengtsson, T., Holefors, A., Witzell, J., Andreasson, E., and Liljeroth, E. 2014a. Activation of defence responses to Phytophthora infestans in potato by BABA. Plant Pathol. 63:193-202.

Bengtsson, T., Weighill, D., Proux-Wéra, E., Levander, F., Resjö, S., Burra, D. D., Moushib, L. I., Hedley, P. E., Liljeroth, E., Jacobson, D., Alexandersson, E., and Andreasson, E. 2014b. Proteomics and transcriptomics of the BABAinduced resistance response in potato using a novel functional annotation approach. BMC Genomics 15:315.

Bilichak, A., Ilnytskyy, Y., Wóycicki, R., Kepeshchuk, N., Fogen, D., and Kovalchuk, I. 2015. The elucidation of stress memory inheritance in Brassica rapa plants. Front. Plant Sci. 6:Article 00005. doi:10.3389/fpls.2015.00005

Bonasio, R. 2015. The expanding epigenetic landscape of non-model organisms. J. Exp. Biol. 218:114-122.

Bonduriansky, R. 2012. Rethinking heredity, again. Trends Ecol. Evol. 27: 330-336.

Bonduriansky, R., Crean, A. J., and Day, T. 2011. The implications of nongenetic inheritance for evolution in changing environments. Evol. Appl. 5:192-201.

Bonduriansky, R., and Day, T. 2009. Nongenetic inheritance and its evolutionary implications. Annu. Rev. Ecol. Evol. Syst. 40:103-125.

Burggren, W. W., and Crews, D. 2014. Epigenetics in comparative biology: Why we should pay attention. Integr. Comp. Biol. 54:7-20.

Danchin, É., Charmantier, A., Champagne, F. A., Mesoudi, A., Pujol, B., and Blanchet, S. 2011. Beyond DNA: Integrating inclusive inheritance into an extended theory of evolution. Nat. Rev. Genet. 12:475-486.

Edmonds, J. M. 1986. Biosystematics of Solanum sarrachoides Sendtner and S. physalifolium Rusby (S. nitidibaccatum Bitter). Bot. J. Linn. Soc. 92:1-38.

English, S., Pen, I., Shea, N., and Uller, T. 2015. The information value of nongenetic inheritance in plants and animals. PLoS One 10:e0116996.

Floryszak-Wieczorek, J., Arasimowicz-Jelonek, M., and Abramowski, D. 2015. BABA-primed defense responses to Phytophthora infestans in the next vegetative progeny of potato. Front. Plant Sci. 6:Article 00844. doi: 10.3389/fpls.2015.00844

Frades, I., Abreha, K. B., Proux-Wera, E., Lankinen, A., Andreasson, E., and Alexandersson, E. 2015. A novel workflow correlating RNA-seq data to resistance levels of wild Solanum species and potato clones to Phytophthora infestans. Front. Plant Sci. 6:Article 00718. doi:10.3389/fpls.2015.00718 
Grönberg, L., Andersson, B., and Yuen, J. E. 2012. Can weed hosts increase aggressiveness of Phytophthora infestans on potato? Phytopathology 102: 429-433.

Hagmann, J., Becker, C., Müller, J., Stegle, O., Meyer, R. C., Wang, G., Schneeberger, K., Fitz, J., Altmann, T., Bergelson, J., Borgwardt, K., and Weigel, D. 2015. Century-scale methylome stability in a recently diverged Arabidopsis thaliana lineage. PLoS Genet. 11:e1004920.

Heard, E., and Martienssen, R. A. 2014. Transgenerational epigenetic inheritance: Myths and mechanisms. Cell 157:95-109.

Henry, E., Yadeta, K. A., and Coaker, G. 2013. Recognition of bacterial plant pathogens: Local, systemic and transgenerational immunity. New Phytol. 199:908-915.

Herrera, C., and Bazaga, P. 2011. Untangling individual variation in natural populations: Ecological, genetic and epigenetic correlates of long-term inequality in herbivory. Mol. Ecol. 20:1675-1688.

Herrera, C. M., Medrano, M., and Bazaga, P. 2014. Variation in DNA methylation transmissibility, genetic heterogeneity and fecundity-related traits in natural populations of the perennial herb Helleborus foetidus. Mol. Ecol. 23:1085-1095.

Holeski, L. M., Jander, G., and Agrawal, A. A. 2012. Transgenerational defense induction and epigenetic inheritance in plants. Trends Ecol. Evol. 27:618-626.

Jablonka, E., and Raz, G. 2009. Transgenerational epigenetic inheritance: Prevalence, mechanisms, and implications for the study of heredity and evolution. Q. Rev. Biol. 84:131-176.

Jisha, K. C., and Puthur, J. T. 2016. Seed priming with BABA ( $\beta$-amino butyric acid): A cost-effective method of abiotic stress tolerance in Vigna radiata (L.) Wilczek. Protoplasma 253:277-289.

Johannes, F., Porcher, E., Teixeira, F. K., Saliba-Colombani, V., Simon, M., Agier, N., Bulski, A., Albuisson, J., Heredia, F., Audigier, P., Bouchez, D., Dillmann, C., Guerche, P., Hospital, F., and Colot, V. 2009. Assessing the impact of transgenerational epigenetic variation on complex traits. PLoS Genet. 5:e1000530.

Kathiria, P., Sidler, C., Golubov, A., Kalischuk, M., Kawchuk, L. M., and Kovalchuk, I. 2010. Tobacco mosaic virus infection results in an increase in recombination frequency and resistance to viral, bacterial, and fungal pathogens in the progeny of infected tobacco plants. Plant Physiol. 153: 1859-1870.

Kawashima, T., and Berger, F. 2011. Green love talks; cell-cell communication during double fertilization in flowering plants. AoB Plants 2011:plr015.

Leimar, O., and McNamara, J. M. 2015. The evolution of transgenerational integration of information in heterogeneous environments. Am. Nat. 185: E55-E69.

Liljeroth, E., Bengtsson, T., Wiik, L., and Andreasson, E. 2010. Induced resistance in potato against Phytophthora infestans - effects of BABA in greenhouse and field tests with different potato varieties. Eur. J. Plant Pathol. 127:171-183.

Luna, E., Bruce, T. J. A., Roberts, M. R., Flors, V., and Ton, J. 2012. Next generation systemic acquired resistance. Plant Physiol. 158:844-853.

Luna, E., van Hulten, M., Zhang, Y., Berkowitz, O., López, A., Pétriacq, P., Sellwood, M. A., Chen, B., Burrell, M., van de Meene, A., Pieterse, C. M. J., Flors, V., and Ton, J. 2014. Plant perception of $\beta$-aminobutyric acid is mediated by an aspartyl-tRNA synthetase. Nat. Chem. Biol. 10:450-456.

Mandal, R., Kathiria, P., Psychogios, N., Bouatra, S., Krishnamurthy, R., Wishart, D., and Kovalchuk, I. 2012. Progeny of tobacco mosaic virusinfected Nicotiana tabacum plants exhibit trans-generational changes in metabolic profiles. Biocatal. Agric. Biotechnol. 1:115-123.
Nandeeshkumar, P., Sarosh, B., Kinia, R., Prakasha, H., and Shettya, H. 2009. Elicitation of resistance and defense related proteins by $\beta$-amino butyric acid in sunflower against downy mildew pathogen Plasmopara halstedii. Arch. Phytopathol. Plant Prot. 42:1020-1032.

Pastor, V., Balmer, A., Gamir, J., Flors, V., and Mauch-Mani, B. 2014. Preparing to fight back: Generation and storage of priming compounds. Front. Plant Sci. 5:Article 00295. doi:10.3389/fpls.2014.00295

Pastor, V., Luna, E., Mauch-Mani, B., Ton, J., and Flors, V. 2013. Primed plants do not forget. Environ. Exp. Bot. 94:46-56.

Reinders, J., Wulff, B., Mirouze, M., Marí-Orodóñez, A., Dapp, M., Bucher, E., Rozhon, W., Bucher, E., Theiler, G., and Paszkowski, J. 2009. Compromised stability of DNA methylation and transposon immobilization in mosaic Arabidopsis epigenomes. Genes Dev. 23:939-950.

Richards, E. J. 2011. Natural epigenetic variation in plant species: A view from the field. Curr. Opin. Plant Biol. 14:204-209.

Roach, D., and Wulff, R. 1987. Maternal effects in plants. Annu. Rev. Ecol. Evol. Syst. 18:209-235.

Shailasree, S., Sarosh, B., Vasanthi, N., and Shetty, H. 2001. Seed treatment with $\beta$-aminobutyric acid protects Pennisetum glaucum systemically from Sclerospora graminicola. Pest Manage. Sci. 57:721-728.

Sharma, A. 2013. Transgenerational epigenetic inheritance: Focus on soma to germline information transfer. Prog. Biophys. Mol. Biol. 113: 439-446.

Shea, N., Pen, I., and Uller, T. 2011. Three epigenetic information channels and their different roles in evolution. J. Evol. Biol. 24:1178-1187.

Slaughter, A., Daniel, X., Flors, V., Luna, E., Hohn, B., and Mauch-Mani, B. 2012. Descendants of primed Arabidopsis plants exhibit resistance to biotic stress. Plant Physiol. 158:835-843.

SPSS. 2014. SPSS Syntax Reference Group. SPSS Inc., Chicago.

Teixeira, F., Heredia, F., Sarazin, A., Roudier, F., Boccara, M., Ciaudo, C., Cruaud, C., Poulain, J., Berdasco, M., Fraga, M., Voinnet, O., Wincker, P., Esteller, M., and Colot, V. 2009. A role for RNAi in the selective correction of DNA methylation defects. Science 323:1600-1604.

van Hulten, M., Pelser, M., van Loon, L. C., Pieterse, C. M. J., and Ton, J. 2006. Costs and benefits of priming for defense in Arabidopsis. Proc. Natl. Acad. Sci. USA 103:5602-5607.

Van Oosten, M. J., Bressan, R. A., Zhu, J.-K., Bohnert, H. J., and Chinnusamy, V. 2014. The role of the epigenome in gene expression control and the epimark changes in response to the environment. Crit. Rev. Plant Sci. 33: 64-87.

Verhoven, K. J., and Gurp, T. P. 2012. Transgenerational effects of stress exposure on offspring phenotypes in apomictic Dandelion. PLoS One 7: e38605.

Walters, D., and Heil, M. 2007. Costs and trade-offs associated with induced resistance. Physiol. Mol. Plant Pathol. 71:3-17.

Walters, D. R., and Paterson, L. 2012. Parents lend a helping hand to their offspring in plant defence. Biol. Lett. 8:871-873.

Youngson, N. A., and Whitelaw, E. 2008. Transgenerational epigenetic effects. Annu. Rev. Genomics Hum. Genet. 9:233-257.

Yu, A., Lepère, G., Jay, F., Wang, J., Bapaume, L., Wang, Y., Abraham, A.-L., Penterman, J., Fischer, R. L., Voinnet, O., and Navarro, L. 2013. Dynamics and biological relevance of DNA demethylation in Arabidopsis antibacterial defense. Proc. Natl. Acad. Sci. USA 110:2389-2394.

Zhang, C., and Hsieh, T.-F. 2013. Heritable epigenetic variation and its potential applications for crop improvement. Plant Breed. Biotechnol. 1: 307-319. 